\title{
Sensibilidade do copépode Nitokra sp. à exposição ao agregado óleo-material particulado em suspensão (OSA)
}

\author{
Ana Carina Matos Silva ${ }^{1^{*}}$ \\ Sanye Soroldoni Guimarães ${ }^{2}$ \\ Olívia Maria Cordeiro de Oliveira ${ }^{1}$ \\ Icaro Thiago Andrade Moreira ${ }^{1}$ \\ Jorge Alberto Trigüis ${ }^{1}$ \\ Manoel Jerônimo Moreira Cruz \\ ${ }^{1}$ Universidade Federal da Bahia (UFBA) \\ Instituto de Geociências (IGEO) \\ Campus de Ondina, \\ Salvador - BA - Brasil. \\ 40170-290 \\ ${ }^{2}$ Universidade Federal do Rio Grande (FURG) \\ Instituto de Oceanografia \\ Campus Carreiros \\ Rio Grande - RS - Brasil \\ 96203-900 \\ anacarinams@gmail.com \\ *Autora correspondente
}

\section{RESUMO}

Em um derramamento de petróleo em zonas de manguezal existe grande possibilidade da agregação do óleo com o material particulado em suspensão, o que poderá determinar o sedimento de fundo como destino final do OSA (Agregado Óleo-Material Particulado em Suspensão) representando um grande risco aos organismos bentônicos. Testes de ecotoxicidade com esses organismos têm sido amplamente utilizados como uma das formas mais precisas de monitoramento biológico aos impactos antrópicos nesse ecossistema. O presente trabalho avalia a ecotoxicidade da exposição ao OSA em sedimentos de manguezal, coletados ao longo do estuário do rio Pardo, município de Canavieiras, Bahia, por meio de ensaios em escala piloto (microescala). O procedimento foi realizado através dos testes toxicológicos de exposição aguda para determinar a $\mathrm{CL}_{50}$ (concentração letal 50\%) utilizando o copépode bentônico Nitokra sp. Para efeito de comparação, foram realizados dois cenários, o primeiro considera a fase aquosa do protocolo de simulação de formação do OSA em microescala, e o segundo, o elutriato a partir de sedimento e óleo homogeneizado em diferentes frações. Foi verificado que a formação do OSA tem potencial muito menos tóxico $\left(\mathrm{CL}_{50} 70,71\right)$ em relação ao elutriato formado a partir dos percentuais de sedimento homogeneizado com óleo cru $\left(\mathrm{CL}_{50} 5,59\right)$ confirmando seu potencial como efetiva forma de limpeza para tratamento de óleo na água.

Palavras-chave: Ecotoxicologia, Agregado Óleo-MPS, Nitokra sp. Geoquímica.

\section{ABSTRACT}

In an oil spill in mangrove areas, there is a great possibility of oil aggregation with suspended particulate matter, which may determine the bottom sediment as the final destination of OSA (Oil-Suspended Particulate Matter), posing a great risk to Benthic organisms. Ecotoxicity tests with these organisms have been widely used as one of the most accurate forms of biological monitoring of the anthropogenic impacts on this ecosystem. The present work evaluates the ecotoxicity of the exposure to OSA in mangrove sediments, collected along the Pardo river estuary, Canavieiras, Bahia, through pilot scale (microscale) trials. The procedure was performed using acute exposure toxicological tests to determine LC50 (lethal concentration 50\%) using the benthic copepod Nitokra sp. For the purpose of comparison, two scenarios were performed, the first considers the aqueous phase of the OSA simulation protocol in microscale, and the second, the elutriate from sediment and oil homogenized in different fractions. It was verified that the formation of the OSA has a much less toxic potential (LC50 70.71) in relation to elutriate formed from the percentages of homogenized sediment with crude oil (LC50 5.59) confirming its potential as an effective form of cleaning for oil treatment in water.

Keywords: Ecotoxicology, Oil-Suspended Particulate Matter (OSA), Nitokra sp., Geochemistry. 


\section{INTRODUÇÃO}

Manguezal é um ecossistema de transição característico de zona de estuário em regiões tropicais e subtropicais, sujeito à influência oceanográfica das mudanças de marés (Santos et al. 2012; Queiroz \& Celino 2008). Nesse ambiente, a granulometria predominantemente fina e argilosa do sedimento proporciona retenção de poluentes, sendo sua toxicidade ampliada afetando a integridade do ecossistema. Os hidrocarbonetos de petróleo destacam-se como compostos capazes de produzir danos imediatos para os organismos e esses efeitos vão depender tanto das características do óleo (tipo, quantidade, qualidade e estado de intemperismo) quanto das variações sazonais das condições climáticas e de maré dominantes (Brito et al. 2009).

Nesse aspecto, destaca-se o Agregado Óleo-Material Particulado em Suspensão (OSA), um complexo que surge da interação entre partículas minerais e óleo em meio aquoso e tem sido amplamente aceito, uma vez que se configura como efetiva forma de limpeza para tratamento de óleo na água (Stoffyn-Egli \& Lee 2002; Khelifa et al. 2007; Wang et al. 2013; Sun \& Zeng 2009). As gotas de óleo são facilmente fragmentadas em tamanhos de poucos micrômetros, o que leva à rápida transferência de manchas de óleo a partir da superfície do mar para a coluna de água, aumentando sua superfície de contato (Le Floch et al. 2002; Stoffyn-Egli \& Lee 2002).

A formação dessas gotículas pode aumentar a concentração de hidrocarbonetos em solução, aumentando também a biodisponibilidade desses produtos, promovendo não só o aumento da degradação natural como também aumento na toxicidade do óleo por conta da exposição de compostos como benzeno,

\section{MATERIAIS E MÉTODOS}

A presente pesquisa utiliza uma metodologia inédita baseada em protocolos amplamente descritos, justificando o detalhamento das atividades executadas. Sua elaboração foi baseada em algumas tolueno e xileno. Esses compostos apresentam considerável solubilidade em água, o que torna os organismos marinhos mais vulneráveis uma vez que absorvem esses contaminantes pelos tecidos, brânquias, por ingestão direta da água ou de alimento contaminado (ITOPF 2013).

O copépodo Nitokra sp. embora não normatizado no Brasil para testes de toxicidade, tem sido amplamente empregado em bioensaios toxicológicos realizados em sedimento por apresentar características peculiares que favorecem sua utilização como organismo-teste. Entre essas características destacam-se o enorme potencial para cultivo em condições laboratoriais, o seu caráter generalista quanto à granulometria do sedimento, constância na sua sensibilidade quanto à substância de referência (Dodecil Sulfato de Sódio-DSS), íntima associação com o ambiente sedimentar, alta abundância e curto ciclo de vida e importância ecológicas elevada. Além disso, representantes com estágios de vida variados podem ser obtidos de cultivos a qualquer época do ano, são fáceis de cultivar em laboratório e requerem espaço mínimo e poucos equipamentos para realização dos testes (Ferraz et al. 2010; Furley et al. 2010; Zaroni et al. 2010; Sousa et al. 2012).

Este artigo tem como objetivo apresentar os resultados das pesquisas que envolveram avaliar a sensibilidade do copépode Nitokra sp. em ensaios toxicológicos agudos com testes estáticos sem renovação, determinando os efeitos adversos da exposição em dois cenários comparativos: (i) a formação do OSA em protocolo experimental em microescala e (ii) a formação do elutriato a partir da homogeneização de frações percentuais de óleo cru e sedimento

etapas das conhecidas metodologias e fazendo adaptações necessárias para obtenção dos resultados.

Foram amostrados sedimentos em seis pontos durante a baixa-mar ao longo do 
Rio Pardo, nas proximidades do município de Canavieiras (Litoral Sul do Estado da Bahia - Brasil), tomando-se o cuidado de coletar os sedimentos nos primeiros cinco centímetros. A coleta dos sedimentos respeitou os limites de um transecto com aproximadamente quatro metros de comprimento onde foram aleatoriamente coletadas 20 porções do substrato de manguezal. Esse material foi homogeneizado em bandeja de vidro e devidamente acondicionado conservandose a temperatura de até $4^{\circ} \mathrm{C}$. Os parâmetros físico-químicos do sedimento $(\mathrm{pH}$, potencial redox, temperatura, salinidade, condutividade, oxigênio dissolvido, sólidos totais e turbidez) foram medidos in situ utilizando uma sonda multiparâmetro (Horiba U-54 Multiparameter Water Quality Checker). Cada amostra de sedimento foi calcinada em mufla a $450{ }^{\circ} \mathrm{C}$, por 6 horas, homogeneizada, peneirada com uma malha de $100 \mu \mathrm{m}$, mantido em geladeira a $4{ }^{\circ} \mathrm{C}$ em recipientes de vidro por um período de 24 horas, até a distribuição do sedimento nos recipientes-testes para o início dos bioensaios, segundo Embrapa (2009).

A caracterização das matrizes óleo e sedimento foram realizados no Laboratório de Estudos do Petróleo (LEPETRO/IGEO/UFBA). As análises de sedimento foram realizadas em um período que não excedeu o prazo de 48 horas após as coletas e incluíram liofilização, peneiramento $(2 \mathrm{~mm})$ e homogeneização das amostras, as análises de granulometria (analisador de partículas com difração a Laser modelo Cilas 1064) de acordo com descrito em Embrapa (2009). Para as simulações foi utilizado óleo cru da bacia de Campos, cuja densidade foi mensurada seis vezes com densitômetro (DMA 5000 Density Meter) a $15^{\circ} \mathrm{C}$. Sua viscosidade também foi determinada três vezes nas mesmas condições de temperatura, através da utilização do viscosímetro rotacional (Haake Viscotester VT500). A distribuição de hidrocarbonetos foi verificada por Cromatografia Gasosa acoplada a um detector por ionização de chama (GC-FID) modelo Varian CP 3800, equipado com coluna capilar modelo DB5 de $60 \mathrm{~m}$ de comprimento, $0,25 \mathrm{~mm}$ de diâmetro interno, fase estacionária de $0,25 \mu \mathrm{m}$ de espessura e fluxo de gás de arraste (hélio).

O cultivo do copépode Nitokra sp. foi realizado em erlenmeyers com capacidade para $1 \mathrm{~L}$, contendo cerca de 750 $\mathrm{mL}$ de água do mar artificial em salinidade 15 tampados com algodão e gaze para não interferir nas trocas gasosas e evitar a evaporação da água. A água salobra artificial em salinidade 15 é obtida através da diluição de $30 \mathrm{~g}$ de sal marinho artificial em $500 \mathrm{~mL}$ de água, e avolumada para um litro. Os cultivos foram mantidos em sala climatizada na temperatura de $25 \pm 1^{\circ} \mathrm{C}$, em estufa com fotoperíodo de $12 \mathrm{~h}$ claro/escuro e sem aeração (Nascimento et al. 2002). Foram utilizados apenas indivíduos adultos (machos ou fêmeas não ovadas) selecionados a partir de uma pequena porção do conteúdo que foi peneirada em malha de $125 \mu \mathrm{m}$, posteriormente contados e separados em estereomicroscópio binocular.

Foram realizados testes toxicológicos agudos em experimentos de simulação, com sedimento e sua fase aquosa considerando dois principais cenários, sem formação do OSA (elutriato oriundo do sedimento misturado ao óleo) e com a formação de OSA (oriunda do experimento de simulação de formação do OSA).

Em paralelo a cada ensaio, foram realizados testes com substância de referência (DSS - $\mathrm{NaC}_{12} \mathrm{H}_{25} \mathrm{SO}_{4}$ ) para elaboração da carta-controle com o objetivo de ilustrar a regularização da sensibilidade do cultivo dos organismos utilizados. Cada ensaio foi acompanhado de análises dos parâmetros físico-químicos associados $(\mathrm{pH}$, oxigênio dissolvido, temperatura e condutividade) no período inicial e final para controlar as condições básicas de exposição e subsidiar a interpretação dos resultados (Nascimento et al. 2002). Foi utilizada a solução-estoque contendo $10 \mathrm{mg}$ de DSS para cada $100 \mathrm{~mL}$ de água salgada em salinidade 15. Cinco concentrações foram testadas, com três réplicas por concentração: 0 (controle), 4, 6, 8, 10 e 12 
mg. $\mathrm{L}^{-1}$. Dez indivíduos foram expostos em cada recipiente contendo $20 \mathrm{~mL}$ da solução de diluição, as condições para o ensaio toxicológico agudo estão descritas no Quadro 1. Após uma exposição de quatro dias, foram contados os organismos vivos e mortos de cada teste com o auxílio de um estereomicroscópio óptico binocular. Foi também realizado um teste agudo com a água do cultivo para certificação da qualidade das culturas (Nascimento et al. 2002).

Os dados produzidos pelos testes toxicológicos foram expressos como dados de mortalidade e sobrevivência dos indivíduos adultos $\left(\mathrm{CL}_{50}\right)$ através do uso do método matemático Probit ou Trimmed Spearman-Karber Method, conforme recomendado pela USEPA (2002), associando a intervalos de confiança de $95 \%$. Os testes com sedimento e contaminante produziram respostas relacionadas à mortalidade (ou sobrevivência) que serão dadas para cada ensaio em comparação com o grupo controle. Verificou-se a intensidade das associações lineares do resultado dos testes com os parâmetros físico-químicos através do Coeficiente de Correlação linear de Pearson considerando $p>0,05$ e para realizar a estimativa da similaridade dos dados, a Análise de Componentes Principais foi utilizada, possibilitando associar os resultados dos parametros ambientais mensurados com os resultados dos ensaios de toxicidade, completando o arcabouço estatístico necessário que se combinam para conduzir o resultado observado.

\subsection{SIMULAÇÃO EM MICROESCALA PARA FORMAÇÃO DO OSA}

$\mathrm{O}$ total de noventa erlenmeyers foram preenchidos com $250 \mathrm{~mL}$ de água salobra artificial em salinidade 15 e pesados $0,175 \mathrm{~g}$ de sedimento para cada um, a fim de respeitar o valor de $0,7 \mathrm{~g} / \mathrm{L}$ de concentração de sedimento, uma vez que segundo Sun \& Zeng (2009) é a concentração máxima para formação de OSA em partículas de até 16 $\mu \mathrm{m}$.

O sedimento foi transferido para os erlenmeyers com a ajuda de um pissete com água salobra artificial. Cada um deles foi tampado com papel alumínio, colocado por um minuto em mesa agitadora e deixado em descanso por uma noite em temperatura de $15^{\circ} \mathrm{C}$ em ambiente escurecido. No dia seguinte, cada um dos erlenmeyers passou mais um minuto em mesa agitadora a $2,1 \mathrm{~Hz}$ (126 RPM) e posteriormente foram adicionados $0,05 \mathrm{~g}\left(50 \mathrm{mg}\right.$ ou $\left.0,05 \mathrm{~cm}^{3}\right) \mathrm{de}$ óleo. Sucedeu-se mais três horas de

\subsection{TESTES TOXICOLÓGICOS AGUDOS}

Para cada ensaio foi utilizada a concentração de OSA de $0,7 \mathrm{~g} / \mathrm{L}$ de sedimento para aproximadamente $0,05 \mathrm{~g}$ de óleo, em sete diluições, com três réplicas para cada diluição, com as seguintes proporções percentuais: $0 \%$ (controle); $1 \%$; $6,25 \% ; 12,5 \% ; 25 \% ; 50 \%$; e $100 \%$. A agitação em mesa agitadora reciprocante a 126 RPM e mais um descanso por uma noite (MOREIRA, 2014). As amostras foram filtradas a vácuo para captação dos agregados formados em filtro de acetato de celulose como descrito por Khelifa e colaboradores (2007) e transferidos dos filtros para frascos de vidro escurecidos.

O método para preparação do elutriato seguiu as normas da USEPA (United States Environmental Protection Agency) descritas no Thecnical Manual (USEPA 2001) combinado uma parte de sedimento em quatro partes de água salobra em salinidade 15. A mistura foi vigorosamente agitada por uma hora em mesa agitadora. $\mathrm{O}$ composto foi centrifugado por 10 minutos a 4.000 RPM com o objetivo de isolar o sedimento da fase aquosa e então foi utilizado imediatamente após o preparo.

parcela aquosa foi adicionada durante o experimento de simulação em microescala de formação do OSA, em frações coletadas na superfície com pipeta automática em diferentes proporções (Tabela 1).

Para o elutriato, oito diluições percentuais foram testadas, com três 
réplicas por concentração, nas seguintes proporções: $0 \%$ (controle); $0,5 \% ; 1 \%$; $6,25 \% ; 12,5 \% ; 25 \%, 50 \%$, 100\%. Dez indivíduos foram adicionados em cada recipiente contendo $15 \mathrm{~mL}$ da solução de diluição do elutriato (Tabela 2). Por questões de resposta dos organismos verificados no teste piloto, foi adicionada a concentração percentual de $0,5 \%$, que compreende o valor intermediário de mortalidade entre a primeira concentração percentual testada e o controle, auxiliando a fidelidade estatística da análise.
A salinidade da água foi monitorada utilizando-se um refratômetro portátil e os parâmetros físico-químicos de todos os testes foram mensurados utilizando um medidor portátil de $\mathrm{pH}$ com precisão de \pm 0.01 unidades de $\mathrm{pH}$; O.D. com um medidor micro processado portátil digital com precisão de $\pm 0,05 \%$; Temperatura com termômetro acoplado ao oxímetro com precisão de $\pm 0,05^{\circ} \mathrm{C}$ e condutividade com condutivímetro, portátil digital com precisão de $\pm 0,05 \%$. As condições para os ensaios de toxicidade aguda estão descritas no Quadro 1

Tabela 1 - Valores utilizados de fase aquosa do ensaio de simulação de formação do OSA e água salobra artificial para testes de toxicidade aguda utilizando o copépode Nitokra sp.

\begin{tabular}{ccc}
\hline Diluições Percentuais & Fase Aquosa $(\mathbf{m L})$ & Água Salobra Artificial $(\mathbf{m L})$ \\
\hline $\mathbf{1 0 0} \%$ & 15 & - \\
$\mathbf{5 0 \%}$ & 7,5 & 7,5 \\
$\mathbf{2 5 \%}$ & 3,75 & 11,25 \\
$\mathbf{1 2 , 5 \%}$ & 1,87 & 13,13 \\
$\mathbf{6 , 2 5 \%}$ & 0,94 & 14,6 \\
$\mathbf{1 \%}$ & 0,15 & 14,85 \\
\hline
\end{tabular}

Tabela 2 - Valores utilizados de elutriato e água salobra artificial para testes de toxicidade aguda utilizando o copépode Nitokra sp.

\begin{tabular}{ccc}
\hline Diluições Percentuais & Elutriato $(\mathbf{m L})$ & Água Salobra Artificial $(\mathbf{m L})$ \\
\hline $\mathbf{1 0 0} \%$ & 15 & - \\
$\mathbf{5 0 \%}$ & 7,5 & 7,5 \\
$\mathbf{2 5 \%}$ & 3,75 & 11,25 \\
$\mathbf{1 2 , 5 \%}$ & 1,87 & 13,13 \\
$\mathbf{6 , 2 5} \%$ & 0,94 & 14,6 \\
$\mathbf{1 \%}$ & 0,15 & 14,85 \\
$\mathbf{0 , 5} \%$ & 0,075 & 14,925 \\
\hline
\end{tabular}

\begin{tabular}{|c|c|}
\hline Parâmetros & Condições \\
\hline Temperatura & $25 \pm 1^{\circ} \mathrm{C}$ \\
\hline Salinidade & 15 \\
\hline Fotoperíodo & Natural (16h claro/8h escuro) \\
\hline Sistema de ensaio & Estático \\
\hline Duração do ensaio & $96 \mathrm{~h}$ \\
\hline Volume da solução-teste & $15 \mathrm{~mL}$ \\
\hline Réplicas por diluição & 3 \\
\hline Organismos por réplica & 10 \\
\hline Idade dos organismos & Adultos \\
\hline Efeito observado & Letalidade (CL50) \\
\hline Validade do ensaio & Mínimo de $70 \%$ de sobrevivência no controle \\
\hline
\end{tabular}




\section{RESULTADOS}

\subsection{CARACTERIZAÇÃO DO ÓLEO E DO SEDIMENTO}

Na Tabela 3 são apresentados os dados de coordenadas geográficas e os parâmetros físico-químicos dos pontos de coleta no substrato de manguezal da região de estudo, estes resultados compõem a necessidade de caracterizar o sedimento utilizado nos ensaios para controle das condições experimentais avaliadas.

As coordenadas estão diretamente relacionadas aos pontos amostrais, como pode ser visto na Tabela 3 , associadas aos horários de coleta nela descritos, os quais obedeceram à restrição de coleta em maré baixa $( \pm 0,3 \mathrm{~m})$.
Os valores de $\mathrm{pH}$ mostraram um caráter neutro a levemente básico (com exceção do ponto 3 que apresentou relativa acidez), com pequena variação entre os seis pontos amostrais, tendo seu máximo registrado no P1 $(9,91)$ e mínimo registrado no P3 $(5,93)$. Foi registrada ampla variação de Eh entre os máximos e mínimos observados nos pontos 3 e $1(325,0 \mathrm{mV})$ e 5 $(45,0 \mathrm{mV})$, respectivamente, sendo que todos demostram significativa característica oxidante. A variação de temperatura não excedeu a diferença de $4,2{ }^{\circ} \mathrm{C}$ entre os pontos, sendo o valor máximo registrado no

Tabela 3 - Coordenadas geográficas e parâmetros físico-químicos descritivos nos pontos de coleta às margens do Rio Pardo no município de Canavieiras, região litoral Sul do estado da Bahia

\begin{tabular}{|c|c|c|c|c|c|c|c|c|c|c|}
\hline \multirow[b]{2}{*}{ Pontos } & \multirow[b]{2}{*}{ Coordenadas } & \multirow[b]{2}{*}{ Hora } & \multicolumn{8}{|c|}{ Parâmetros físico-químicos (in situ) } \\
\hline & & & $\mathbf{p H}$ & $\begin{array}{c}\mathbf{E h} \\
(\mathrm{mV})\end{array}$ & $\begin{array}{c}\text { Temp. } \\
\left({ }^{\circ} \mathrm{C}\right)\end{array}$ & Sal. & $\begin{array}{l}\text { Condt. } \\
(\mathrm{S} / \mathrm{m})\end{array}$ & $\begin{array}{c}\text { O.D. } \\
(\mathrm{mL} / \mathrm{L})\end{array}$ & $\begin{array}{l}\text { S.T.D. } \\
(\mathrm{mg} / \mathrm{L})\end{array}$ & $\begin{array}{c}\text { Turbidez } \\
\text { (NTU) }\end{array}$ \\
\hline $\mathrm{P} 1$ & $\begin{array}{c}\text { S } 15^{\circ} 41^{\prime} 58^{\prime \prime} \\
\text { W } 38^{\circ} 55^{\prime} 54^{\prime \prime}\end{array}$ & $07: 35$ & 9,91 & 45 & 26,56 & 30 & 30,8 & 15,2 & 17,4 & 121,0 \\
\hline $\mathrm{P} 2$ & $\begin{array}{l}\mathrm{S} 15^{\circ} 41^{\prime} 36^{\prime \prime} \\
\text { W } 38^{\circ} 56^{\prime} 21^{\prime \prime}\end{array}$ & $08: 57$ & 8,55 & 163 & 29,62 & 20 & 35,7 & 18,3 & 21,8 & 26,6 \\
\hline $\mathrm{P} 3$ & $\begin{array}{l}\text { S } 15^{\circ} 40^{\prime} 52^{\prime \prime} \\
\text { W } 38^{\circ} 56^{\prime} 24^{\prime \prime}\end{array}$ & $09: 22$ & 5,93 & 325 & 30,02 & 12 & 23,4 & 17,66 & 14,5 & 30,6 \\
\hline P4 & $\begin{array}{l}\text { S } 15^{\circ} 41^{\prime} 26^{\prime \prime} \\
\text { W } 38^{\circ} 57^{\prime} 05^{\prime \prime}\end{array}$ & $10: 03$ & 7,89 & 245 & 30,82 & 6 & 15,5 & 21,04 & 9,61 & 49,1 \\
\hline P5 & $\begin{array}{l}\text { S } 15^{\circ} 41^{\prime} 25^{\prime \prime} \\
\text { W } 38^{\circ} 57^{\prime} 35^{\prime \prime}\end{array}$ & $10: 56$ & 7,61 & 240 & 30,37 & 6 & 14,8 & 19,63 & 9,16 & 99,2 \\
\hline P6 & $\begin{array}{l}\text { S } 15^{\circ} 40^{\prime} 58^{\prime \prime} \\
\text { W } 38^{\circ} 58^{\prime} 25^{\prime \prime}\end{array}$ & $11: 47$ & 7,91 & 222 & 30,08 & 5 & 12,3 & 18,95 & 7,62 & 179 \\
\hline
\end{tabular}

Legenda: $\mathrm{pH}$ = Potencial Hidrogeniônico; $\mathrm{Eh}=$ Potencial de Redução; Temp. $=$ Temperatura; Sal. = Salinidade; Condt. $=$ Condutividade; O.D. = Oxigênio Dissolvido; S.T.D. = Sólidos Totais Dissolvidos; NTU = Unidades Nefelométricas de Turbidez

P4 $\left(30,8^{\circ} \mathrm{C}\right)$ e o valor mínimo visto no P1 $\left(26,5^{\circ} \mathrm{C}\right)$. A salinidade, conforme esperado para um ambiente de estuário, se manteve relativamente alta nos três primeiros pontos $(30,20,12)$ e reduziu significativamente nos três últimos (6, 6 e 5 , respectivamente). Para a condutividade, foi observada ampla variação com destaque para o valor máximo apresentado no P2 (35,7 mS). Os valores de oxigênio dissolvido variaram entre $\mathrm{o}$ máximo de $21,04 \mathrm{~mL} / \mathrm{L}$ e o mínimo de $15,20 \mathrm{~mL} / \mathrm{L}$. O maior valor encontrado em sólidos totais dissolvidos foi visto no P2 $(21,8 \mathrm{mg} / \mathrm{L})$ e o menor valor no P6 (7,6 $\mathrm{mg} / \mathrm{L})$. O maior valor de turbidez foi encontrado no P6 (179,0 NTU) seguido do P1 (121,0 NTU) e o menor valor foi registrado no P2 (20,6 NTU).
Cada um destes valores evidencia um conjunto de condições ambientais da área de estudo que não só possibilitaria, mas atenuaria a formação do OSA nestes locais, considerando os fatores que influenciam na formação destes agregados em ambiente natural.

Com relação ao perfil granulométrico dos pontos de coleta, as médias para as proporções encontradas nas análises mostram uma predominância das frações consideradas como areia muito fina $(62,5-125 \mu \mathrm{m})$ e silte $(3,9-62,5 \mu \mathrm{m})$ (Figura 1) segundo a classificação granulométrica proposta pela escala de Krumbein (1951). 


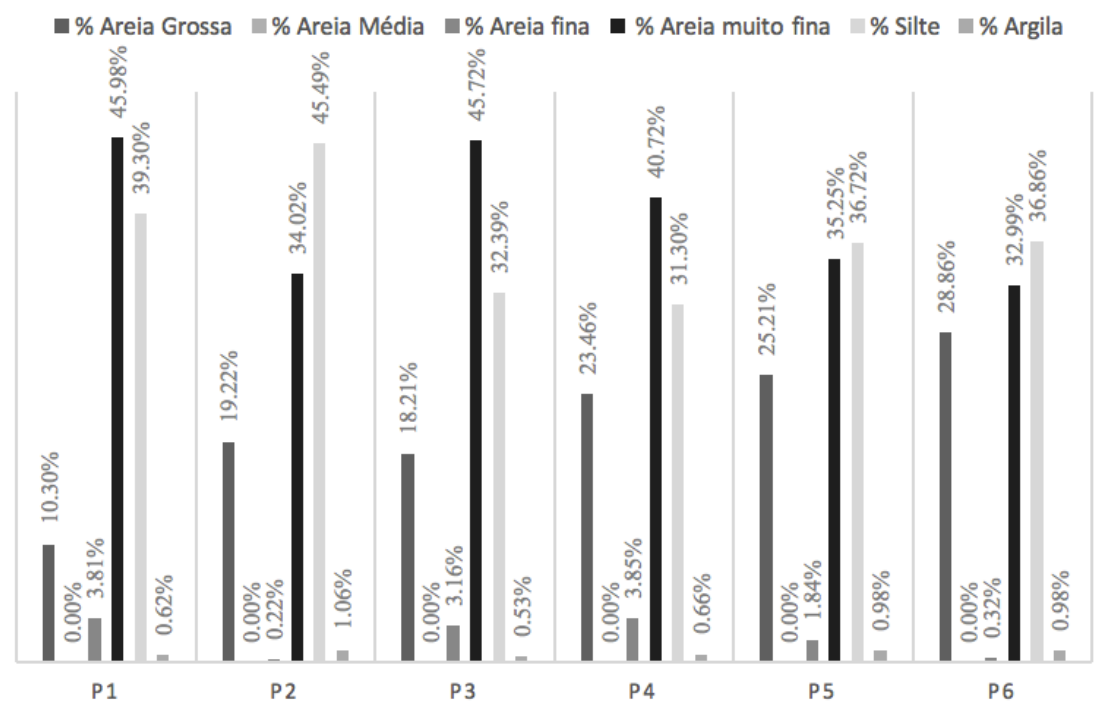

Figura 1 Representação gráfica dos valores percentuais das frações granulométricas de sedimento ao longo do Rio Pardo no município de Canavieiras, região litoral Sul do estado da Bahia, nos diferentes pontos amostrais.

O óleo cru da bacia de Campos utilizado para a realização dos testes apresentou densidade de $0,8823 \mathrm{~g} / \mathrm{mL}$, a média da viscosidade de $33,44 \mathrm{mPa} \cdot \mathrm{s}$. Com relação a distribuição de hidrocarbonetos, $\mathrm{o}$ cromatograma apresentou uma maior composição de n-alcanos de baixo peso molecular (nC10-nC17), sugerindo óleos tipicamente marinhos (Figura 2).

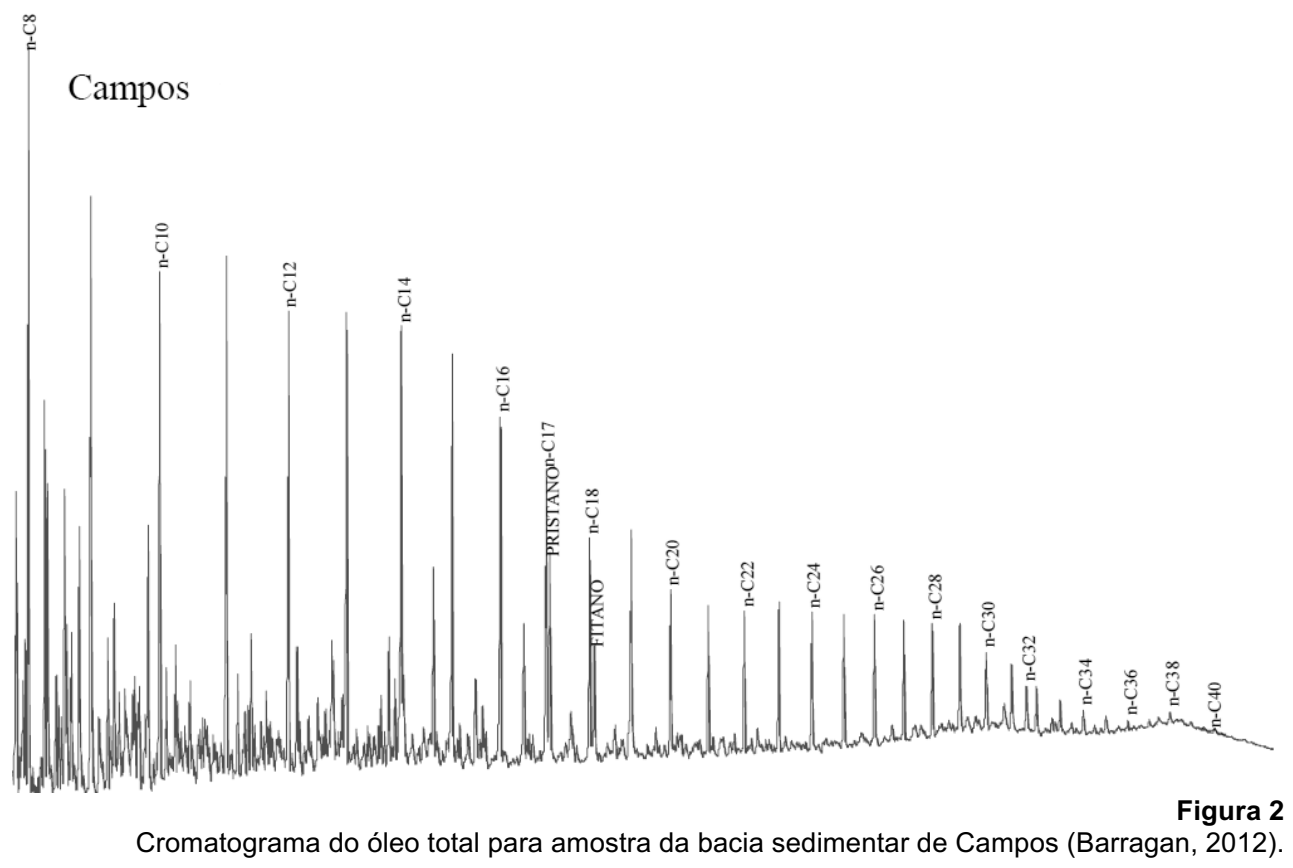

\subsection{TESTES COM SUBSTÂNCIA DE REFERÊNCIA COM DSS}

Os testes de toxicidade aguda com a substância de referência DSS apresentaram resultados dentro dos limites de aceitabilidade descritos $(<9,25 \mathrm{mg} / \mathrm{L})$ (Cariello 2012). Os valores de $\mathrm{CL}_{50}-96 \mathrm{~h}$ foram de 4,66; 5,73 e 4,38 mg/L, estando, portanto, dentro da faixa de sensibilidade estabelecida. A Tabela 4 especifica os valores de $\mathrm{CL}_{50}$ encontrados para cada teste, bem como os intervalos de confiança (95\%), e os parâmetros físico-quimicos mensurados. 
Tabela 4 - Valores de $\mathrm{CL}_{50}$ com os limites de confiança (95\%) e parâmetros físico-químicos representativos dos testes de referência com DSS seguidos da Média, Desvio Padrão e Coeficiente de Variância

\begin{tabular}{cccccc}
\hline Teste de referência (DSS) & $\mathbf{C L}_{\mathbf{5 0}} \mathbf{( m g / L )}$ & Limite de Confiança & pH & O.D. & Temperatura ( $\left.{ }^{\circ} \mathbf{C}\right)$ \\
\hline TR1 & 4,66 & $3,83-5,67$ & 7,05 & 6,79 & 24,1 \\
TR2 & 5,73 & $4,69-6,98$ & 7,03 & 6,85 & 25,2 \\
TR3 & 4,38 & $3,51-5,46$ & 7,66 & 6,53 & 25,8 \\
Média & 4,92 & $* *$ & 7,25 & 6,72 & 25,03 \\
Desvio padrão & 0,71 & $* *$ & 0,36 & 0,17 & 0,86 \\
Coef. Vari. & 0,14 & $* *$ & 0,05 & 0,03 & 0,03 \\
Legenda: TR = Teste de Referência; ** = Não se aplica & & &
\end{tabular}

\subsection{TESTES TOXICOLÓGICOS AGUDOS}

Os resultados do teste de toxicidade com a fase aquosa de formação do OSA e seus ajustes realizados através do uso dos métodos estatísticos Probit e Trimmed Spearman-
Karber estão dispostos na Tabela 5 e posteriormente, na Tabela 6 , são descritos os parâmetros físico-químicos registrados no início e ao final das análises.

Tabela 5 - Dados percentuais de mortalidade e sobrevivência dos testes de toxicidade agudos a partir da fase aquosa de simulação da formação do OSA utilizados para determinar a $\mathrm{CL}_{50}$ incluindo proporções ajustadas para cálculo e limite de confiança $(95 \%)$

\begin{tabular}{cccc}
\hline Concentração & Mortalidade & Sobrevivência & Proporções ajustadas \\
\hline $\mathbf{0 \%}($ controle) & 0,33 & 9,67 & 0,0300 \\
$\mathbf{1 \%}$ & 2,00 & 8,00 & 0,1658 \\
$\mathbf{6 , 2 5 \%}$ & 2,33 & 7,67 & 0,2002 \\
$\mathbf{1 2 , 5 \%}$ & 2,33 & 7,67 & 0,2002 \\
$\mathbf{2 5 \%}$ & 2,67 & 7,33 & 0,2357 \\
$\mathbf{5 0 \%}$ & 4,33 & 5,67 & 0,4330 \\
$\mathbf{1 0 0 \%}$ & 6,00 & 4,00 & 0,6000 \\
\hline $\mathbf{C L}_{\mathbf{5 0}} \mathbf{( \% )}$ & & 70,71 & $33,9-151,9$ \\
\hline
\end{tabular}

Tabela 6 - Parâmetros físico-químicos iniciais e finais representativos do teste de toxicidade aguda com o copépode Nitokra sp. a partir da fase aquosa de simulação da formação do OSA, seguidos da Média, Desvio Padrão e Coeficiente de Variância

\begin{tabular}{|c|c|c|c|c|c|c|c|c|}
\hline \multirow[b]{2}{*}{ Conc. (\%) } & \multicolumn{2}{|c|}{ pH } & \multicolumn{2}{|c|}{ O.D. (mg/L) } & \multicolumn{2}{|c|}{ Temperatura $\left({ }^{\circ} \mathrm{C}\right)$} & \multicolumn{2}{|c|}{ Condutividade $(\mu \mathrm{S})$} \\
\hline & Inicial & Final & Inicial & Final & Inicial & Final & Inicial & Final \\
\hline $0 \%$ & 7,15 & 6,85 & 6,74 & 5,5 & 23,5 & 23,8 & 23,8 & 21,85 \\
\hline $1 \%$ & 7,10 & 7,05 & 6,6 & 5,78 & 23,8 & 24 & 20,49 & 21,97 \\
\hline $6,25 \%$ & 7,15 & 7,1 & 6,48 & 5,99 & 24 & 24,2 & 21,32 & 20,76 \\
\hline $12,50 \%$ & 7,15 & 7,2 & 6,37 & 5,57 & 24 & 23,6 & 20,95 & 23,21 \\
\hline $25 \%$ & 7,12 & 7,05 & 6,03 & 5,37 & 24,5 & 23,3 & 22,05 & 22,63 \\
\hline $50 \%$ & 7,15 & 6,75 & 5,54 & 5,68 & 24,6 & 23,3 & 20,15 & 22,49 \\
\hline $100 \%$ & 7,1 & 7,45 & 5,32 & 5,72 & 25,5 & 23 & 22,49 & 21,49 \\
\hline MÉD & 7,13 & 7,11 & 5,815 & 5,585 & 24,65 & 23,3 & 21,41 & 22,455 \\
\hline DP & 0,0244 & 0,2926 & 0,4743 & 0,1567 & 0,6245 & 0,2449 & 1,0606 & 0,7148 \\
\hline $\mathrm{CV}$ & 0,003 & 0,0411 & 0,0815 & 0,0280 & 0,0253 & 0,0105 & 0,0495 & 0,0318 \\
\hline
\end{tabular}

Os resultados do teste de toxicidade com o elutriato produzido a partir da matriz de sedimento e óleo homogeneizado e suas correções estão dispostos na Tabela 7 e na
Tabela 8, subsequentemente, os parâmetros físico-químicos registrados no início e ao final das análises. 
Tabela 7 - Dados percentuais de mortalidade e sobrevivência dos testes de toxicidade agudos a partir do elutriato, utilizados para determinar a $\mathrm{CL}_{50}$ incluindo proporções ajustadas para cálculo e limite de confiança (95\%)

\begin{tabular}{cccc}
\hline Concentração & Mortalidade & Sobrevivência & Proporções ajustadas \\
\hline $\mathbf{0 \%}($ controle) & 1,67 & 8,33 & 0,000 \\
$\mathbf{0 , 5 \%}$ & 2,67 & 7,33 & 0,0641 \\
$\mathbf{1 \%}$ & 2,67 & 7,33 & 0,0641 \\
$\mathbf{6 , 2 5 \%}$ & 5,33 & 4,67 & 0,4037 \\
$\mathbf{1 2 , 5 \%}$ & 5,67 & 4,33 & 0,4471 \\
$\mathbf{2 5 \%}$ & 7,00 & 3,00 & 0,6170 \\
$\mathbf{5 0 \%}$ & 10,00 & 0,00 & 1,0000 \\
$\mathbf{1 0 0 \%}$ & 10,00 & 0,00 & 1,0000 \\
\hline $\mathbf{C L}(\mathbf{5 0})$ & & 5,59 & $5,59-2,87$ \\
\hline
\end{tabular}

Pode-se afirmar de acordo com os dados observados, que a Concentração Letal (CL) que afeta $50 \%$ dos organismos da espécie Nitokra sp. para exposição no elutriato é de 5,59\%, demonstrando alta toxicidade, e para exposição ao OSA essa concentração é de $70,71 \%$, o que significa uma toxicidade menor para o organismo em questão.

Tabela 8 - Parâmetros físico-químicos iniciais e finais representativos do teste de toxicidade aguda com o copépode Nitokra sp. a partir do elutriato, seguidos da Média, Desvio Padrão e Coeficiente de Variância

\begin{tabular}{ccccccccc}
\hline \multicolumn{2}{c}{$\mathbf{p H}$} & \multicolumn{3}{c}{ O.D. $(\mathbf{m g} / \mathbf{L})$} & \multicolumn{2}{c}{ Temperatura $\left({ }^{\circ} \mathbf{C}\right)$} & \multicolumn{2}{c}{ Condutividade $(\boldsymbol{\mu S})$} \\
\hline Conc. & Inicial & Final & Inicial & Final & Inicial & Final & Inicial & Final \\
\hline $\mathbf{0 \%}$ & 5,95 & 6,15 & 4,9 & 4,53 & 25,1 & 23,9 & 32,66 & 23,95 \\
$\mathbf{0 , 5 \%}$ & 5,95 & 5,65 & 5,25 & 5,88 & 24,3 & 22,2 & 29,82 & 29,26 \\
$\mathbf{1 \%}$ & 6,05 & 6,05 & 5,65 & 4,77 & 24,7 & 24,5 & 27,24 & 29,76 \\
$\mathbf{6 , 2 5 \%}$ & 6,05 & 5,9 & 4,72 & 4,53 & 24,5 & 24,1 & 24,98 & 23,18 \\
$\mathbf{1 2 , 5 0 \%}$ & 5,85 & 5,55 & 4,31 & 4,65 & 25,7 & 24,5 & 23,8 & 24,28 \\
$\mathbf{2 5 \%}$ & 5,95 & 5,45 & 4,8 & 4,19 & 25,4 & 25,5 & 23,4 & 21,22 \\
$\mathbf{5 0 \%}$ & 7,15 & 6,75 & 5,54 & 5,68 & 24,6 & 23,3 & 20,15 & 22,49 \\
$\mathbf{1 0 0} \%$ & 7,1 & 7,45 & 5,32 & 5,72 & 25,5 & 23 & 22,49 & 21,49 \\
$\mathbf{M E ́}$ & 6,5125 & 6,3 & 4,9925 & 5,06 & 25,3 & 24,075 & 22,46 & 22,37 \\
$\mathbf{D P}$ & 0,7087 & 0,9678 & 0,5507 & 0,7627 & 0,4830 & 1,1500 & 1,6346 & 1,3856 \\
$\mathbf{C V}$ & 0,1088 & 0,1536 & 0,1103 & 0,1507 & 0,0191 & 0,0478 & 0,0728 & 0,0619 \\
\hline
\end{tabular}

Legenda: Conc. $=$ Concentração; O.D. $=$ Oxigênio Dissolvido.

Verificou-se a intensidade das associações lineares do resultado dos testes com os parâmetros físico-químicos através do Coeficiente de Correlação linear de Pearson. Os valores marcados em negrito mostraram significância para correlação considerando $p>0,5$. Esta análise foi utilizada para verificação dos parâmetros que mais contribuem para toxicidade, sendo encontradas fortes correlações positivas entre a diluição percentual e a mortalidade, e inversa para o oxigênio dissolvido (Tabela 9).

Tabela 9 - Matriz de correlação de Pearson para parâmetros físico-químicos e ensaios de toxicidade com o copépode Nitokra sp.

\begin{tabular}{|c|c|c|c|c|c|c|}
\hline & Conc. & Mort. & pH & O.D. & Temp. & Condt. \\
\hline D.P. & 1,00 & & & & & \\
\hline Mort. & 0,94 & 1,00 & & & & \\
\hline pH & 0,47 & 0,47 & 1,00 & & & \\
\hline O.D. & $-0,87$ & $-0,81$ & $-0,19$ & 1,00 & & \\
\hline Temp. & 0,72 & 0,82 & 0,61 & $-0,42$ & 1,00 & \\
\hline Condt. & 0,00 & $-0,29$ & 0,03 & $-0,19$ & $-0,52$ & 1,00 \\
\hline
\end{tabular}


Foi utilizada a Análise de Componentes Principais (ACP) para realizar a estimativa da similaridade dos dados, representando-os em duas dimensões ortogonais. Os dois primeiros fatores, representados na Figura 3 explicam a maior proporção da variância total entre todas as combinações lineares dos dados originais, tendo a segunda menor proporção de variância total que a primeira e assim sucessivamente.

A análise dos componentes principais permitiu a representação dos dados em uma área bivariada (Figura 3). Os dois eixos principais - Fator 1 e 2, explicam $83,19 \%$ da variância total $(60,66 \%$ e $22,53 \%$ ), representados principalmente pelas variáveis mortalidade, concentração, temperatura, oxigênio dissolvido (fator 1) e condutividade (fator 2).

Nessa representação gráfica foi possível corroborar a relação negativa entre o oxigênio dissolvido e os outros parâmetros estudados, verificada através da correlação de Pearson, e a forte correlação positiva entre os fatores de toxicidade (concentração e mortalidade) sendo o pH o menos influente entre os parâmetros físicoquímicos monitorados.

Com relação aos fatores de explicabilidade do teste, os autovalores (eigenvalues) representam a razão da variação entre os grupos pela variação dentro dos mesmos, considerando que quanto mais distante de 1 , maior será a variação entre os grupos explicada pela função discriminante.

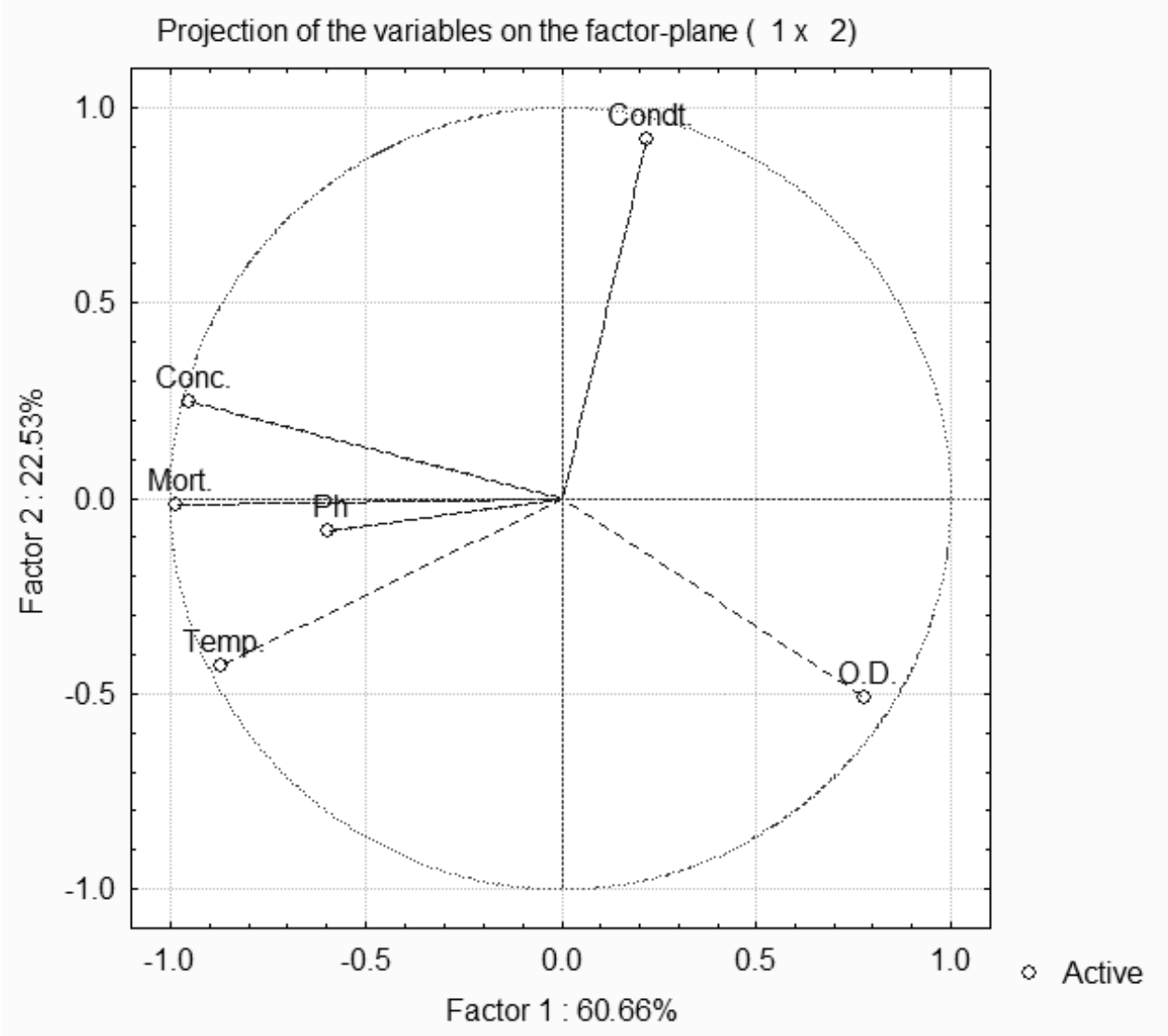

Figura 3 
Tabela 10 - Autovalores (eigenvalues), percentual total de variância e acumulados referentes aos fatores de explicabilidade encontrados para a matriz de correlação

\begin{tabular}{lcccc}
\hline & Autovalores & \% total da variância & Autovalor acumulado & \% acumulada \\
\hline Componente 1 & 3,639330 & 60,65551 & 3,639330 & 60,6555 \\
Componente 2 & 1,351569 & 22,52615 & 4,990899 & 83,1817 \\
Componente 3 & 0,863636 & 14,39394 & 5,854536 & 97,5756 \\
Componente 4 & 0,098554 & 1,64257 & 5,953090 & 99,2182 \\
Componente 5 & 0,046837 & 0,78061 & 5,999927 & 99,9988 \\
Componente 6 & 0,000073 & 0,00122 & 6,000000 & 100,0000 \\
\hline
\end{tabular}

Através da análise da Tabela 10, é possível discriminar os seis principais fatores que contribuem para o total da variância entre os parâmetros, sendo a primeira componente a que tem efetivamente o maior poder de separação intergrupal.

\section{DISCUSSÕES}

Os parâmetros físico-químicos descritivos apresentaram comportamento dentro do esperado para ambiente característico de manguezal. A baixa variação nos valores de $\mathrm{pH}$ demonstra indício de uniformidade de características entre esses locais, consequência de contribuições semelhantes das águas marinhas e doce nos pontos amostrais. Em contraste, verificou-se ampla variação de Eh, que representa a instabilidade deste ambiente durante a medição deste parâmetro, mantendo sua significativa característica oxidante (Queiroz \& Celino 2008). A variação de temperatura (não superior a $4,2^{\circ} \mathrm{C}$ entre os pontos) pode estar relacionada à influência das condições climáticas em função dos horários e locais de coleta, integradas às características intrínsecas dos pontos amostrados.

Com relação ao observado para a salinidade, há uma redução gradual nos valores, onde os maiores índices foram encontrados em pontos amostrais que sofrem influência da salinidade do aporte marinho à jusante e os menores à montante do rio (Figura 4.4). Esse comportamento deve-se à penetração da maré, que faz com que as amostras coletadas em manguezais que apresentam proximidade maior à foz do estuário tenham salinidades mais elevadas.

Os parâmetros $\mathrm{pH}$, Eh, e salinidade são dependentes da temperatura, aumentando e diminuindo de acordo com a variação desta, de forma não linear. Todos esses fatores, juntos ou isoladamente contribuem para que os cátions que se encontram sorvidos às partículas que constituem os substratos dos manguezais possam tornar-se biodisponíveis (Queiroz \& Celino 2008), portanto, os resultados dos parâmetros físico-químicos nas diferentes estações amostrais são determinantes para que se possa correlacionar seus possíveis efeitos como influentes para a relação de toxicidade encontrada nos sedimentos.

O oxigênio dissolvido é uma variável que sofre influência da composição dos sedimentos, da temperatura, da salinidade da água e de fatores hidrodinâmicos e oferece indicativo acerca das condições naturais do ambiente e possibilita detectar possíveis impactos ambientais como a eutrofização e a poluição orgânica. Gás carbônico, oxigênio molecular, íons de nitrito e nitrato e a própria água são as principais fontes para o oxigênio dissolvido (Baumgarten et al. 1996).

É possível observar que o oxigênio dissolvido teve um padrão comportamental de valores muito semelhante à temperatura. Os valores de temperatura nos diferentes pontos podem ser considerados um fator determinante para a distribuição dos valores de oxigênio dissolvido, visto que a temperatura determina a solubilidade dos gases (Fiorucci, 2005). Foi também verificada uma relação inversa entre o comportamento do Eh comparado à condutividade, visto que a presença de íons dissolvidos pode exercer enorme influência 
nos valores de condutividade elétrica registrados (Queiroz \& Celino 2008).

Os valores de sólidos totais foram diretamente proporcionais aos encontrados para a condutividade. Sampaio e colaboradores (2007) já haviam desenvolvido estudos que demonstravam que existe uma forte correlação linear entre esses dois parâmetros, que pode ser explicada pela razão destes estarem em solução e em estado coloidal (Braile \& Cavalcanti 1993). Segundo Pina e colaboradores (2003) a elevada turbidez do estuário é consequência do elevado escoamento de maré, da carga fluvial, da grande extensão de áreas intertidais e ainda da grande susceptibilidade para a geração de ondas que intensificam a ressuspensão.

Considerando que, de acordo com Sun e Zeng (2009), a granulometria é um fator determinante para formação e destino dos OSAs formados e que a formação do OSA é atenuada em sedimento com granulometria de tamanho inferior a $5 \mu \mathrm{m}$ em função de sua maior área de superfície de contato, a predominância da fração silte no perfil granulométrico das amostras confirma a alta viabilidade para formação de OSA na área de estudo considerada.

O valor da relação Pristano/Fitano $(\mathrm{Pr} / \mathrm{Ph})>1$ encontrada para o óleo da Bacia de Campos é indicador de um ambiente de deposição com condições oxidantes (óxidas). A relação $\mathrm{nC} 17 / \mathrm{nC} 29>1$ (nC29 é um indicador terrestre e $\mathrm{nC} 17$ é um indicador de material marinho de algas), indica origem predominantemente de aporte de material marinho. As relações $\mathrm{Pr} / \mathrm{nC} 17 \mathrm{e}$ $\mathrm{Ph} / \mathrm{nC} 18$ menores que 1 , e o valor de $\mathrm{IPC}=1,07$, são indicativos de maturidade para esse óleo (Barragan 2012). Não foram observadas para o óleo evidencias de biodegradação, justificando seu uso para os testes realizados.

A correlação negativa entre o O.D. e os fatores de toxicidade (concentração e mortalidade), pode ser explicada porque o oxigênio dissolvido apresenta depleção relacionada principalmente à decomposição de matéria orgânica e respiração dos organismos, embora alguns compostos como a amônia aumentem sua toxicidade em baixas concentrações de oxigênio, tendo como consequência mecanismos fisiológicos compensatórios a fim de minimizar os efeitos da hipóxia nesses organismos (Amaral 2012). Além disso, há uma menor contribuição desse fator para aumento da toxicidade, visto que a aeração não é uma exigência no cultivo destes organismos e que, por conta destes fazerem uso do oxigênio nos seus processos respiratórios, pode causar uma redução da sua concentração no meio e a depender da magnitude, o consequente aumento da toxicidade (Metcalf \& Eddy 2003).

De acordo com Jiang e colaboradores (2010), a fração de óleo acomodada na água demonstra alta toxicidade para o zooplancton marinho, principalmente nos primeiros estágios de vida, visto que compostos como o HPA e o benzeno podem se acumular nos tecidos devido às suas habilidades lipofílicas. Em algumas espécies, uma tendência à tolerância pode se manifestar em diferentes estágios de vida e a resistência a toxicidade aos compostos do óleo pode ser variada.

É importante destacar que, mesmo considerando a exposição ao experimento de simulação de formação de OSA, onde foi observado um baixo valor de $\mathrm{CL}_{50}(5,59)$, demostrando um potencial letal menos tóxico, diversos estudos evidenciam os efeitos à exposição em baixa concentração para o zooplancton, como anormalidades comportamentais na alimentação (Jensen et al. 2008; Calbet et al. 2007; Barata et al. 2002), aumento no consumo de oxigênio (Smith e Hargreaves 1984), crescimento e desenvolvimento anormal, e diminuição da taxa de crescimento e (Bellas \& Thor 2007; Bejarano et al. 2006; Olmstead \& Leblanc 2005). 


\section{CONCLUSÕES}

Concluiu-se que, em salvaguardo de suas diferentes características intrínsecas, a porção aquosa concentrada da simulação mostrou-se consideravelmente menos tóxica (CL50 70,71\%) do que o elutriato formado a partir das diluições percentuais do sedimento com óleo (CL50 5,59\%).

Com relação ao poder explicativo da influência das variáveis monitoradas, destacam-se as correlações fortes e positivas entre os fatores de toxicidade (concentração e mortalidade) em detrimento do oxigênio dissolvido, que demonstrou correlação forte e negativa ao

\section{AGRADECIMENTOS}

Esse artigo foi financiado pela Coordenação de Aperfeiçoamento de Pessoal de Nível Superior (CAPES) apoiado pela Universidade Federal da Bahia (UFBA), com apoio logístico e metodológico de campo do Núcleo de Estudos Ambientais (NEA), e pela

\section{REFERÊNCIAS}

Amaral K. G. C. 2012. Correlação entre fator de toxicidade e parâmetros físico-químicos para efluentes domésticos tratados. Dissertação de Mestrado, Universidade Tecnológica Federal do Paraná. Curitiba. 105 p.

Barata C., Baird D., Medina M., Albalat A., Soares A. M. 2002. Determining the ecotoxicological mode of action of toxic chemicals in meiobenthic marine organisms: stage-specific short tests with Tisbe battagliai. Marine Ecology Progress Series, 230: 183-194.

Barragan O. L. V. 2012. Caracterização geoquímica de óleos da América Latina. Dissertação de Mestrado, Instituto de Geociências, Universidade Federal da Bahia. Salvador. 109 p.

Baumgarten M. G. Z., Rocha J. M. B., Niencheski L. F. H. 1996. Manual de análises em oceanografia química.. Editora da FURG. Rio Grande. 132 p.

Bejarano A. C., Chandler G. T., He L. 2006. Individual to population level effects of South Louisiana crude oil water accommodated hydrocarbon fraction (WAF) on a marine meiobenthic copepod. Journal of Experimental Marine Biology and Ecology, 332: 49-59. passo em que o $\mathrm{pH}$ pareceu ser o fator menos determinante para o resultado dos testes dentre as variáveis físico-químicas avaliadas neste estudo.

Dada à complexidade e a variabilidade dos hidrocarbonetos e compostos insolúveis em água, é necessário destacar a importância da relação das análises de toxicidade juntamente com o monitoramento dos parâmetros físicoquímicos, uma vez que os limites de confiança dos efeitos não são muito pequenos.

Fundação Universidade Federal do Rio Grande (FURG), com apoio logístico e metodológico de laboratório pelo Laboratório de Microcontaminantes Orgânicos e Ecotoxicologia Aquática (CONECO).

Bellas J., Thor P. 2007. Effects of selected PAHs on reproduction and survival of the calanoid copepod Acartiatonsa. Ecotoxicology, 16: 465474.

Braile P. M. \& Cavalcanti J. E. W. A. 1993. Manual de tratamento de águas residuárias industriais. São Paulo: CETESB, 764 p.

Brito S. E. M., Duran R., Guyoneaud R., GoñiUrriza M., Oteyza T. G., Crapez M. A. C., Aleluia I., Wasserman J. C. A. 2009. A case study of in situ oilcontamination in a mangrove swamp (Rio De Janeiro, Brazil). Marine Pollution Bulletin, 58: 418-423.

Calbet A, Saiz E., Barata C. 2007. Lethal and sublethal effects of naphthalene and 1, 2dimethylnaphthalene on the marine copepod Paracartiagrani. Marine Biology, 151: 195-204.

Cariello M. S. 2012. Efeito do Naftaleno na microalga marinha Dunaliellatertiolecta, ouriçodo-mar Lytechinusvariegatus, e nos microcrustáceos estuarinos Nitokrasp. e Leptocheirusplumulosus. Dissertação de Mestrado, Universidade de São Paulo, 135 p. 
EMBRAPA Centro Nacional de Pesquisa de Solos. 2009. Manual de análises químicas de solos, plantas e fertilizantes. Silva F. C. Campinas: Embrapa Informática Agropecuária. Rio de Janeiro: Embrapa Solos, 370 p.

Ferraz M. A., Zaroni L. P., Bergmann Filho T. U., Gasparro M. R., Sousa E. C. P. M. 2010. Taxa de eclosão de ovos do copépodo Nitokra sp. em diferentes salinidades. In: Congresso Brasileiro De Ecotoxicologia, 11, Anais. Bombinhas, Santa Catarina.

Fiorucci A. R. 2005. A importância do oxigênio dissolvido em ecossistemas aquáticos. Química Nova na Escola, 22:10 - 16.

Furley T. H., Vitali M. M., Sobreira R. G., Zaroni L. P. 2010. Sensibilidade aguda do copépodo Nitokra sp. ao sulfato de zinco em diferentes salinidades. In: Congresso Brasileiro De Ecotoxicologia, 11, Anais. Bombinhas, Santa Catarina.

ITOPF. 2013. Fate of Marine Oil Spills: Technical Information Paper. London, UK. n 2, 2011. Disponível em: $<$ http://www.itopf.com/informationservices/publications/documents/ tip2fateofmarineoilspills.pdf $>$. Acesso em $10 \mathrm{dez}$ 2016.

Jensen M. H., Nielsen T. G., Dahllöf I. 2008. Effects of pyrene on grazing and reproduction of Calanusfinmarchicus and Calanusglacialis from Disko Bay, West Greenland. Aquat. Toxicol., 87: 99-107.

Jiang Z., Huang Y., Xu S., Liao Y., Shou L., Liu J., Chen Q., Zeng J. 2010. Advance in the toxic effects of petroleum water accommodated fraction on marine plankton. Acta Ecol. Sin., 30:8-15.

Khelifa A., Fieldhouse B., Pjontek D., Wang Z., Yang C., Landriault M., Fingas M., Brown C. E., Gamble L. 2007. A Laboratory Study on Formation of Oil-SPM Aggregates using the NIST Standard Reference Material 1941b. In: Proceedings Of The Thirtieth Arctic And Marine Oil Spill Program Technical Seminar, Environment Canada, Ottawa, Ontario, 1, p. 3548.

Krumbein W. C. \& Sloss L. L. 1951. Stratigraphy and sedimentation. Soil Science, 71:401.

Le Floch S., Guyomarch J., Merlin F. X., StofynEgli P., Dixon J., Lee K. 2002. The influence of salinity on oil-mineral aggregate formation. Spill science e technology bulletin, 8:65-71.

Metcalf \& Eddy, Burton F. L., Stensel H. D., Tchobanoglous G. 2003. Wastewater engineering: treatment and reuse. 4 ed. Boston: McGraw-Hill. 1408 p.

Moreira I. T. A. 2014. Investigação de possíveis impactos ecológicos do petróleo sobre comunidades biológicas estuarinas na Baía de
Todos os Santos e no Sul da Bahia: OSA como uma ferramenta norteadora. Tese de Douturado, Instituto de Geociências, Universidade Federal da Bahia, Salvador. 265 p.

Nascimento I. A., Sousa E. C. P. M., Nipper M. 2002. Métodos em ecotoxicologia marinha: aplicações para o Brasil. 262 p.

Olmstead A. W. \& Leblanc G. A. 2005. Joint action of polycyclic aromatic hydrocarbons: predictive modeling of sublethal toxicity. Aquat. Toxicol., 75:253-262.

Pina P., Braunschweig F., Saraiva S., Santos M., Martins F., Neves R. 20003. A importância dos Processos Físicos no controlo da Eutrofização em Estuários. Sapientia. Algarve. Disponível em $<$ http://hdl.handle.net/10400.1/40>. Acesso em: 04 abr. 2012.

Queiroz A. F. S. \& Celino J. J. 2008. Manguezais e Ecossistemas estuarinos na Baía de Todos os Santos. In: Queiroz, A. F. De S.; Celino, J. J. Avaliação de ambientes na Baía de Todos os Santos: aspectos geoquímicos, geofísicos e biológicos. Salvador - BA: EDUFBA, p. 39-58.

Sampaio S. C., Silvestro M. G., Frigo E. P., Borges C. M. 2007. Relação entre série de sólidos e condutividade elétrica em diferentes águas residuárias. Irriga, 12:557-562.

Santos L. C., Cunha-Lignon M., Schaeffer-Novelli Y., Citrón-Morelo G. 2012. Long-term effects of oil pollution in mangrove forests (Baixada Santista, Southeast Brazil) detected using a GISBased multitemporal analysis of aerial photographs. Brazilian Journal of Oceanography, 60:161-172.

Smith R. L., Hargreaves B. R. 1984. Oxygen consumption in Neomysis Americana (Crustacea:Mysidacea) and the effects of naphthalene exposure, Mar. Biol., 79:109-116.

Sousa E. C. P. M., Zaroni L. P., Filho T. U. B., Marconato A. A., Kirschbaum A. A., Gasparro M. R. 2012. Acute sensitivity to Nitokra sp benthic copepod to potassium dichromate and ammonia chloride. J. Braz. Soc. Ecotoxicol., 7: 75-81.

Stoffyn-Egli P. \& Lee K. 2002. Formation and characterization of oil-mineral aggregates. Spill science and technology bulletin, 8:31 - 44.

Sun J. \& Zheng X. 2009. A review of oil suspended particulate matter aggregation a natural process of cleansing spilled oil in the aquatic environment. Journal of environmental monitoring: JEM, 11:1801-1809.

UNITED STATES ENVIRONMENTAL PROTECTION AGENCY - USEPA. 2002. Testing Manual EPA-821-R-02-012 - Methods for measuring the acute toxicity of effluents and receiving waters to freshwater and marine organisms. Out. 2002. Disponível em: < http://water.epa.gov/scitech/methods/cwa/wet/u 
pload/2007_07_10_methods_wet_disk2_atx16.pdf $>$. Acesso em 10 Jan 2014.

\section{UNITED STATES ENVIRONMENTAL} PROTECTION AGENCY - USEPA. 2001. Testing Manual EPA-823-B-01-002 - Methods for collection, storage, and manipulation of sediments for chemical and toxicological analyses. Oct 2001. Disponível em: < http://water.epa.gov/polwaste/sediments/cs/uplo ad/collectionmanual.pdf >. Acesso em $04 \mathrm{Fev}$ 2014.
Wang W., Zheng Y., Lee K. 2013. Chemical dispersion of oil with mineral fines in a low temperature environment. Marine Pollution Bulletin, 72:205 - 2012.

Zaroni L. P., Bergmann F. T. U., Ferraz M. A., Gasparro M. R., Sousa E. C. P. M. 2010. Taxa de eclosão de ovos do copépodoNitokra $\mathrm{sp}$. em diferentes tipos de sedimento. In: Congresso Brasileiro De Ecotoxicologia, 11, Anais. Bombinhas, Santa Catarina. 